•科普・

\title{
家庭小实验揭示炒青菜裉绿之谜
}

姜雅茹§, 赵亮 ${ }^{\S}$, 赵卫光 ${ }^{*}$, 关英 ${ }^{*}$

南开大学化学学院, 天津 300371

摘要: 从科学的角度解释了炒青菜时经常会出现的青菜变色现象。为什么大多数蔬菜是绿色的? 在加热过程中青菜 经历了哪些生理和化学变化? 生活中常用哪些方式保持炒青菜时不变色? 本文讨论了这些问题及其背后的科学道 理, 同时设计了可在家中完成的实验进行科学验证, 结果表明在焯水的过程中加入适量的盐和油, 炒时不盖锅盖能 很好地保持青菜原本的颜色。

关键词: 家中实验室; 绿色蔬菜; 叶绿素

中图分类号: G64; O6

\section{Uncover the Mystery of Green Fading While Cooking Vegetables in Home Lab}

Yaru Jiang ${ }^{\S}$, Liang Zhao ${ }^{\S}$, Weiguang Zhao *, Ying Guan *

College of Chemistry, Nankai University, Tianjin 300071, P. R. China.

Abstract: The phenomenon of green fading while cooking vegetables is explained scientifically in this paper. Why most vegetables are green? What kind of physiological and chemical changes occurs to the vegetables during heating? How to prevent the green fading while cooking vegetables in daily life? All these questions are discussed in detail. A series of scientific experiments in home lab are also designed to keep green while cooking vegetables. The results indicate that vegetables might keep green via pretreatment with proper amount of salt or oil in the boiling water, or cooking without the lid covering the pot.

Key Words: Home laboratory; Green vegetables; Chlorophyll

作为指导教师, 我们在《大学化学》曾发表了题为 “有机化学实验教学对科研素养的培养一 兴趣、家中实验室与化学思维培养” 的教学改革论文 ${ }^{[1]}$, 介绍了利用家中实验室培养本科生的有机 化学实验兴趣、化学思维以及科研素养的作用。本文是学生自发地利用课余时间撰写的蔬菜在烹饪 过程中为什么会变色的科普文章, 并利用家中的条件设计实验, 对蔬菜烹饪过程中的 “保绿” 方法 进行了科学的验证和分析。文章和实验均出自学生之手，我们对文章进行了适当的修订。

\section{1 古老国度的青菜历史}

20 世纪 50 年代, 考古人员在半坡遗址出土的陶罐中发现了白菜和芥菜种子, 说明我国种植蔬 菜的历史已有六七千年之久 ${ }^{[1]}$ 。其实早在新石器时代, 人们就开始采摘食用野菜, 如考古学家从浙

收稿: 2020-07-02; 录用: 2020-07-20; 网络发表：2020-08-12 $\S$ 共同第一作者, 南开大学化学学院 2018 级本科生

*通讯作者, Email: yingguan@nankai.edu.cn; zwg@nankai.edu.cn

基金资助：南开大学 2020 年本科教育教学改革一流课程建设项目(NKJG2020115) 
江吴兴钱山漾遗址中发掘出的菱角和甜瓜子, 还有浙江余姚河姆渡遗址的菱角和瓠 ${ }^{[3]}$ 。大约从那时 起，中国的饮食文化就拉开了序幕吧。

从 “食不厌精, 脍不厌细” 的春秋时期开始到清朝结束, 两千多年的时间, 我国的蔬菜品种发 展至 100 多种。智慧的炎黄子孙在发展饮食文化的道路上从未止步, 从最原始的烤多烹杄, 到后来 的水煮、油制烹饪 ${ }^{[4]}$ ，青菜的烹调方法越来越多样化，满足了人们对美食的不断追求。

人们常说 “色香味” , 最能勾起人们对美食的欲望的就是见到 “她” 的第一眼, 那种怦然心动 的感觉总能激起人们的食欲。然而, 我们在家炒菜时为什么很难保持那诱人的绿色呢? 蔬菜内部又 发生了哪些奇妙的故事呢?

\section{2 蔬菜为什么大多是绿色的?}

\section{1 叶绿素在哪里?}

我们日常食用的蔬菜大多数是绿色的, 这是因为蔬菜中含有一种叫做叶绿素的物质。叶绿素究 竟分布在哪里呢？我们要从植物细胞的结构说起。

如果我们把植物细胞比作工业园区, 那么植物细胞的细胞壁和细胞膜便是园区的两道围墙, 它 们共同控制着物质的进出; 园区里面各栋厂房就是植物细胞的细胞器, 它们分别是叶绿体、线粒体、 内质网、高尔基体、液泡等; 而细胞核则是 “细胞园区” 的主管，它控制着整个园区的正常运作。

使植物显绿色的物质主要集中在叶绿体这间 “工厂” 内，它也是植物的 “养料制造车间” 和 “能 量转换站” (图 1A)。叶绿体的外膜和内膜构成了厂房的 “围墙”，他们控制特定的 “人员” 和 “物资” 的进出。工厂里充满了基质, 叶绿体、DNA、各种酶类都在这里忙碌, 光合作用的重要原料一二氧 化碳也存放在这里。工厂里还有许许多多的 “反应釜”, 我们称之为类囊体, 他们像硬币一样堆叠起 来, 形成了我们熟悉的基粒。叶绿素就在类囊体膜上, 类囊体腔里面则住着质子、 $\mathrm{NADP}^{+}$(烟酰胺腺 嘌呤二核苷磷酸)等。每到白天，他们就忙着利用二氧化碳和水合成淀粉。
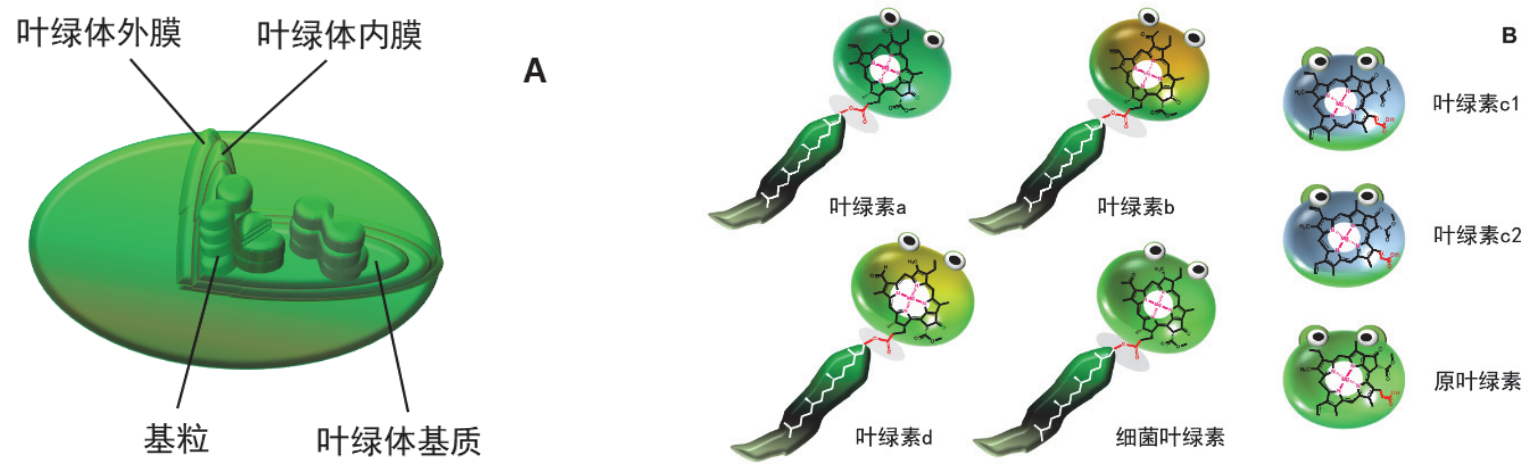

图 1 叶绿体和叶绿素的结构

(A) 叶绿体的结构; (B) 叶绿素的结构

\section{2 叶绿素的结构特点}

叶绿素的结构十分特别, 像一个可爱的小蝌蚪(图 1B)。亲水的 “头部” 有个好听的名字叫卟啉 环, 它通过卟啉环中单键和双键的改变就能把可见光一口一口地吃下去。额, 它可是有 “脑子” 的 吆, 那就是卟啉环中央镁离子, 镁离子带正电荷, 与其相连的氮原子则偏向于带负电荷, 因而卟啉 具有极性, 是亲水的, 可以与蛋白质结合 ${ }^{[5]}$ 。“尾巴” 则是一个长长的疏水脂肪烃, 叫做叶绿醇(也叫 植醇)。叶绿醇决定了叶绿素的脂溶性, 主要负责将叶绿素 “固定” 在膜上。叶绿醇在动物体内的氧 化代谢不仅能为动物提供能量, 还可以作为信号分子参与糖脂代谢和脂肪细胞分化调控过程 ${ }^{\left[{ }^{6}\right.}$ 。对 了, 不要忘记小蝌蚪也是有脖子的, 叫做酯键, 通过它把 “头部” 和 “尾巴” 结构连起来。 
究竟为什么叶绿素是绿色的呢? 我们都知道, 大部分有机化合物的颜色是由本身吸收或反射的 可见光的颜色所决定的。可见光主要包括红、橙、黄、绿、蓝、靛、紫七种颜色, 用可见光照射有 机化合物, 其分子结构中不同类型的电子发生从低能级向高能级的跃迁, 吸收相应频率的光能, 不 吸收或者反射什么波长的光, 它便呈现该波长对应的颜色, 如果反射全波长的光, 它就显示白色。 我们将叶绿素对可见光的吸收情况绘制在一张图中, 便可得到叶绿素的 “彩色照片” ——紫外-可见 吸收光谱。图 2 中内嵌的小图是叶绿素 $\mathrm{a}$ 和 $\mathrm{b}$ 的完整吸收谱图, 大图列出了各种叶绿素吸收可见光 时的 “偏好”。可以看出, 由于大部分的叶绿素主要喜欢 “食用” 蓝紫光和红光, 而几乎不 “食用” 绿光, 所以导致叶绿素呈现绿色。这也就是大多数蔬菜都是绿色的原因。

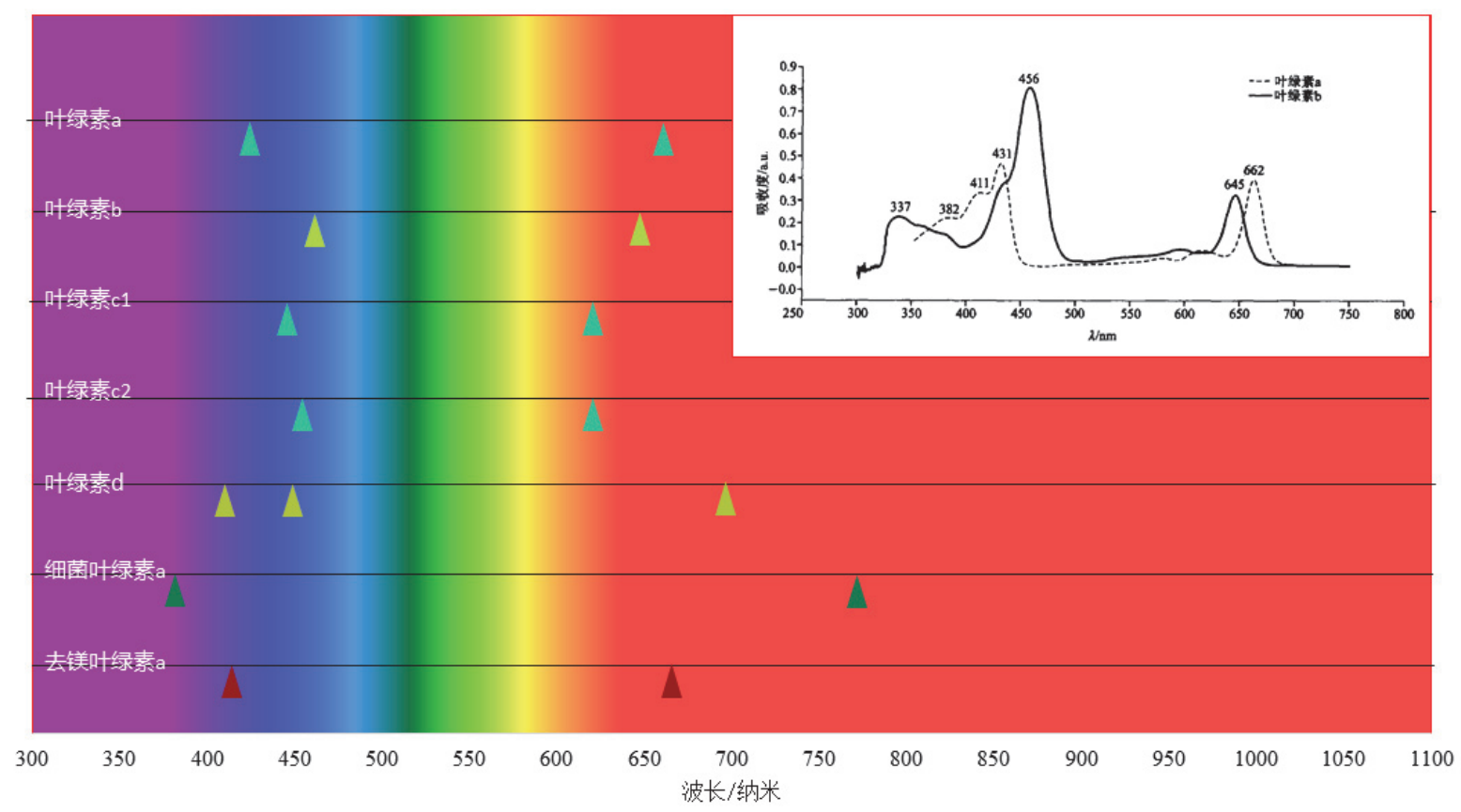

图 2 叶绿素在丙酮溶液中的最大吸收峰波长和叶绿素 $\mathbf{a} 、 \mathbf{b}$ 的紫外-可见吸收光谱(内嵌小图)

最大吸收峰波长数据引自文献 ${ }^{[7,8]}$, 其中细菌叶绿素 $\mathrm{a}$ 的最大吸收峰波长为在乙醇溶液中测得;

叶绿素 $a 、 b$ 的紫外-可见吸收光谱图取自文献 ${ }^{[9]}$

大家千万别以为只有植物当中含有叶绿素。事实上, 可以进行光合作用的生物都含有叶绿素, 它们兄弟众多, 各个都非常漂亮(图 1B)。大哥叶绿素 $\mathrm{a}$ 稳重, 是蓝绿色, 只存在于植物中; 二哥叶 绿素 $\mathrm{b}$ 活泼, 是黄绿色, 在高等植物、绿藻和眼虫藻中都有存在; 叶绿素 $\mathrm{c}$ 是双胞胎, 和大哥一样, 为蓝绿色, 存在于硅藻、鞭毛藻和褐藻中。而叶绿素 $\mathrm{d}$ 和二哥一样, 为黄绿色, 存在于红藻中。此 外, 在黑暗环境中生长的黄化植物中有大量的原叶绿素, 原叶绿素是无色的, 见到阳光它就可以变 成叶绿素了; 在进行光能自养的紫色细菌中也发现了叶绿素的存在, 我们称之为细菌叶绿素 ${ }^{[7]}$, 不 过在藻类和细菌中通常只含有叶绿素而没有叶绿体。叶绿素颜色的不同主要是和他们的结构有关, 例如叶绿素 $\mathrm{a}$ 和 $\mathrm{b}$ 的分子结构中唯一的差异是在头部相同位置的取代基不同, $\mathrm{a}$ 是甲基, $\mathrm{b}$ 是醛基, 取代基不同导致卟啉环上电子云密度变化，所以叶绿素 $\mathrm{a}$ 是绿色，叶绿素 $\mathrm{b}$ 是黄绿色。

\section{3 炒青菜褪绿之谜}

前面提到过, 细胞膜就是保护细胞的围墙, 膜结构的成分主要是磷脂、蛋白质和多糖等。磷脂 
和叶绿素一样, 也是有头有尾的蝌蚪结构, 它们尾部朝内、头部朝外, 组成膜的基本骨架一一磷脂 双分子层。蛋白质或者附着在其表面, 或者嵌入其中, 与磷脂一起, 形成膜的 “流动镶嵌” 结构。 随着炒菜温度升高, 细胞膜内靠氢键、范德华力等非化学键维持的蛋白质空间结构被破坏, 导致膜 结构出现大量的漏洞和空隙; 同时组成细胞膜骨架的磷脂分子热运动越来越剧烈, 形成了其他形式 的聚集体, 如囊泡结构, 导致细胞膜的磷脂双分子层破裂; 最终无情地将 “围墙” 拆出一个个大洞, 使得叶绿素不得不走上 “背井离乡路”。

此外, 叶绿素也是很娇气的(图 3)。首先, 叶绿素在叶绿素酶的作用下, 酯键很容易被弄断, 植 醇尾巴离开了, 孤零零的脑袋就是我们熟知的叶绿酸, 或称之为 “脱植醇叶绿素”, 它仍然是绿色 的。其次, 叶绿素受热或遇酸是不稳定的, 镁卟啉环中的 $\mathrm{Mg}^{2+}$ 很容易被 $\mathrm{H}^{+}$取代, 形成脱镁叶绿素, 没有大脑的叶绿素就变成黄褐色了, 这是由于 $\mathrm{Mg}^{2+}$ 被 $\mathrm{H}^{+}$取代后, 卟啉环上的电子云密度下降, 以 叶绿素 $\mathrm{a}$ 为例, 脱美前后其蓝光区的最大吸收峰波长 $\left(\lambda_{\max }\right)$ 从 $430 \mathrm{~nm}$ 蓝移到 $406 \mathrm{~nm}$ (图 2) ${ }^{[7]}$ 。接着, 脱镁叶绿素的甲酯基受热也会脱落, 形成焦脱镁叶绿素, 颜色进一步加深, 变成暗绿色的了 [10]。同 样, 脱植醇叶绿素也可以发生脱镁和脱甲酯基的反应, 生成脱植醇脱镁叶绿素和脱植醇焦脱镁叶绿 素, 它们的颜色分别是黄褐色和暗绿色。告诉你一个小秘密, 叶绿素很容易被其他金属离子 “洗脑”, 如被换了 “脑子” 的铜代叶绿素颜色更加鲜艳 ${ }^{[11]}$, 作为色素被广泛使用, 在医学和光电转换方面也 有应用呢。

下面我们再来描述一下烹调过程中叶绿素是怎样 “遭遇不测” 的。炒菜刚开始时, 温度还不是 很高, 在叶绿素酶和 $\mathrm{H}^{+}$的作用下, 酯键断裂, 形成叶绿酸和植醇。随着温度升高, 一方面, 叶绿素 和叶绿酸的头部发生脱镁、脱甲酯基反应, 分别生成黄褐色和暗绿色的产物, 导致蔬菜褪绿。另一 方面, 细胞膜结构也会被破坏, 原来被 “封装” 的叶绿素有机会和植物体内的有机酸接触, 同时还 会受到光和氧气的作用。前者进一步促进了叶绿素的脱镁和水解反应, 后者会使叶绿素分解成一系 列小分子物质而褪色, 光氧化产物是乳酸、柠檬酸、琥珀酸、马来酸以及少量丙氨酸。此外, 叶绿 素及其降解产物也会因为细胞膜的破裂而逃逸到汤汁中，导致蔬菜的褪色。

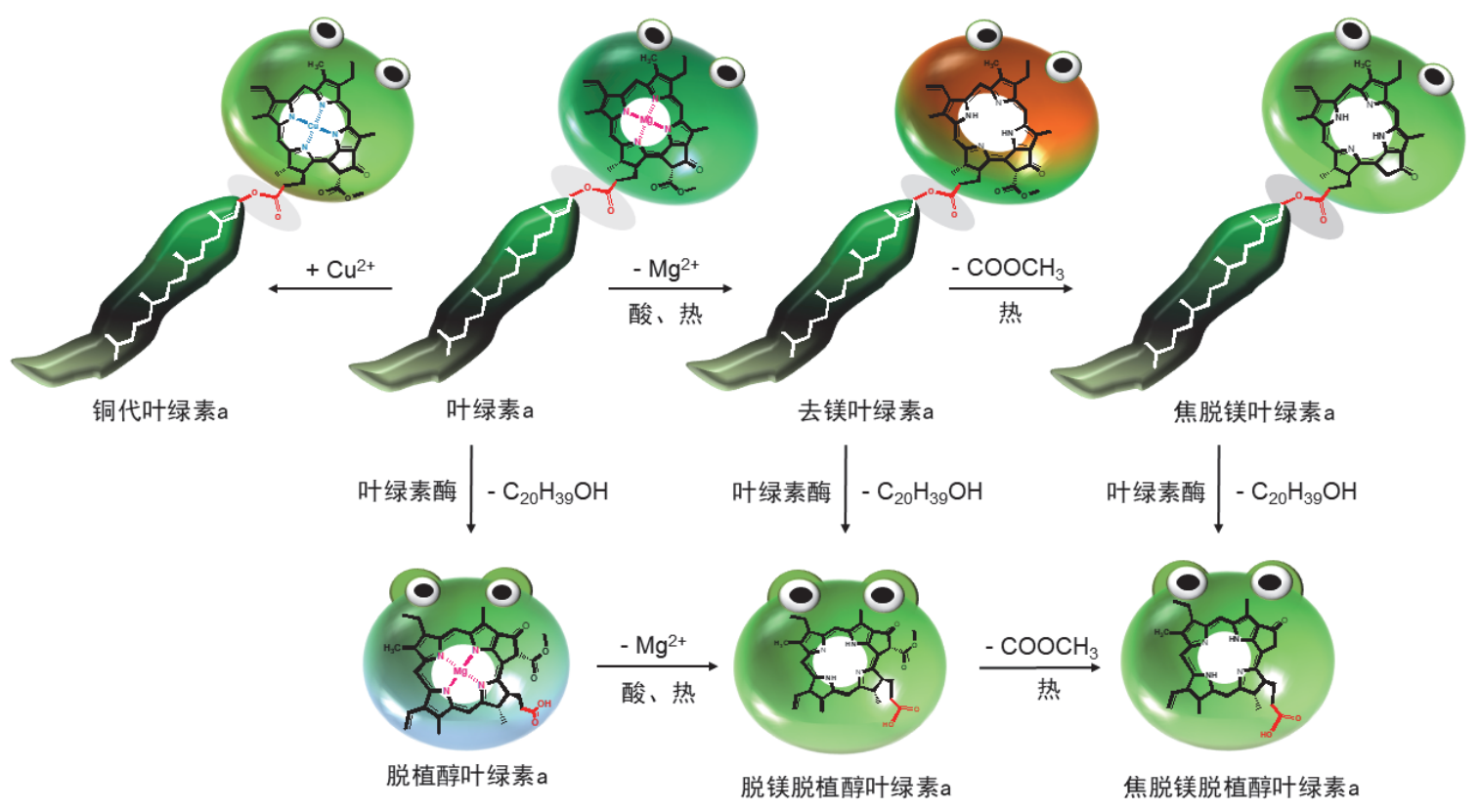

图 3 叶绿素及其衍生物之间的转化 (以叶绿素 $\mathbf{a}$ 为例) 


\section{4 “保绿”小倥门}

看到这里, 大家应该能猜到几个 “保绿” 的小倥门了吧, 下面我们谈谈如何保护好叶绿素兄弟。

\section{1 选用新鲜蔬菜 ${ }^{[12]}$}

俗话说: “烂菜一筐不如青葱一条”。蔬菜的放置时间对蔬菜的新鲜度有很大的影响。刚刚采摘 的蔬菜由于植物细胞仍处于活性状态, 叶绿素还来不及转变成脱镁叶绿素等衍生物。蔬菜在一段时 间内仍呈鲜绿色。但随着放置时间的增加, 脱离土壤的蔬菜没有了水分和养料的来源, 当细胞储存 的养分消耗殆尽后, 叶绿素被叶绿素酶分解, 或经光氧化而漂白, 蔬菜里的花青素和类胡萝卜素显 露出来, 蔬菜就变黄了, 大部分营养物质也被消耗。秋天, 树叶黄了、枫叶红了也是这个道理。因 此, 选用新鲜采摘、存储期短的蔬菜是个前提。

\section{2 炒前焯水}

将蔬菜提前进行焯水处理能够最大程度的保护叶绿素不被破坏。焯时最好要确保大火沸水快速 焯水, 有经验的厨师还会在焯水前在水中加入少量盐和油。加入油是因为油在翻滚的沸水中会分散 成小油滴, 包裹在蔬菜外层, 一定程度上阻滞了水和蔬菜的接触进而减少了水溶性营养物质的损失, 还起到隔绝空气, 降低光线和氧气对蔬菜的光氧化作用。加入食用盐则是构建渗透压平衡体系, 使 植物细胞处于细胞内外渗透压相近的环境中, 减少因渗透和扩散作用而导致的营养物质流失。这里 需要注意的是焯水时尽量不要把蔬菜切得太碎, 否则会导致水溶性的维生素 $\mathrm{C}$ 大量流失, 使我们无 法在日常饮食当中补充到足够的维生素 C。

\section{3 不要盖锅盖}

俗话说 “加火不如加盖”, 尽管这可以让食物熟得快, 可殊不知, 这样炒出来的菜色并不美观。 蔬菜中含有有机酸, 不同蔬菜有机酸种类也不同, 常见为草酸、乙酸、丙二酸等。不盖锅盖一方面 有利于有机酸的挥发, 降低其与叶绿素作用程度, 保持蔬菜翠绿颜色; 另一方面也利于除去对人体 有害的挥发性有机酸。

\section{4 加碱}

为了构建弱碱性环境, 有时也会在焯水时加入少量食用碱, 弱碱性条件下, 叶绿素水解成叶绿 醇、叶绿酸。叶绿酸的化学性质比叶绿素稳定很多, 这也会使蔬菜能够保持鲜绿色。

\section{5 教你在家科学做实验}

说了这么多, 还差一句最重要的话 “实践出真知”, 接下来让我们以厨房为战场, 以炒锅为武器, 用科学的方法在家一起验证一下蔬菜 “保绿”的方法。

\section{1 实验原料与器具}

原料: $900 \mathrm{~g}$ 菠菜; $900 \mathrm{~g}$ 油菜; 精制盐; 花生油; 食用碱面。

器具: 厨房秤; 电炒锅; 家用炒勺; 家用菜盘。

\section{2 操作步骤}

首先将青菜用清水洗净, 选用不新鲜的青菜 $100 \mathrm{~g}$ (提前室温下放置 $48 \mathrm{~h}$ )作为对照组, 编为 1

号。挑选绿度相近的菜叶分成 7 份, 每份净重 $100 \mathrm{~g}$, 依次编号为 $2,3,4 \cdots \cdots .8$ 号。

处理过程为: 将 $1 \mathrm{~L}$ 水烧至 $100^{\circ} \mathrm{C}$, 按照表 1 加入相应的物质后, 加入相应的食材, 焯 $1 \mathrm{~min}$ 后 立即捞出, 放入冷水待用。焯水后以相同的加热挡位、翻炒方式和翻炒时间对全部食材进行处理。 样品的预处理条件和炒菜条件汇总见表 1 。

这里我们没有测试加碱和加油盐同时处理的条件, 原因是碱和油会发生皇化反应, 另外碱也含 有钠离子，可以保持渗透压。 
表 1 蔬菜焯水和炒制时的处理方法和平均 $\boldsymbol{G}$ 值

\begin{tabular}{|c|c|c|c|c|c|c|c|c|}
\hline \multirow{2}{*}{$\begin{array}{l}\text { 条件 } \\
\text { 组号 }\end{array}$} & \multirow{2}{*}{$\begin{array}{c}\text { 蔬菜 } \\
\text { 是否新鲜 }\end{array}$} & \multicolumn{4}{|c|}{ 焯水 } & \multirow{2}{*}{$\begin{array}{c}\text { 炒时是否敞 } \\
\text { 开锅盖 }\end{array}$} & \multicolumn{2}{|c|}{ 平均 $G$ 值 } \\
\hline & & $\begin{array}{c}\text { 是否加油 } \\
(5 \mathrm{~g})\end{array}$ & $\begin{array}{c}\text { 是否加盐 } \\
(2 \mathrm{~g})\end{array}$ & $\begin{array}{c}\text { 是否加油盐 } \\
(5 \mathrm{~g}+1 \mathrm{~g})\end{array}$ & $\begin{array}{c}\text { 是否加碱 } \\
(1 \mathrm{~g})\end{array}$ & & 菠菜 & 油菜 \\
\hline $0^{\text {a }}$ & $\times$ & & & - & & & 78.2 & 72.1 \\
\hline $0^{\text {a }}$ & $\sqrt{ }$ & & & - & & & 87.5 & 98.0 \\
\hline 1 & $x$ & $\times$ & $\times$ & $x$ & $\times$ & $\times$ & 23.0 & 35.4 \\
\hline 2 & $\sqrt{ }$ & $\times$ & $x$ & $\times$ & $\times$ & $\times$ & 46.8 & 55.2 \\
\hline 3 & $\sqrt{ }$ & $\sqrt{ }$ & $x$ & $\times$ & $\times$ & $\times$ & 48.2 & 53.0 \\
\hline 4 & $\sqrt{ }$ & $\times$ & $\sqrt{ }$ & $x$ & $\times$ & $\times$ & 54.4 & 67.4 \\
\hline 5 & $\sqrt{ }$ & $\times$ & $x$ & $\sqrt{ }$ & $\times$ & $\times$ & 66.4 & 76.6 \\
\hline 6 & $\sqrt{ }$ & $\times$ & $\times$ & $x$ & $\times$ & $\sqrt{ }$ & 56.0 & 63.0 \\
\hline 7 & $\sqrt{ }$ & $\times$ & $x$ & $x$ & $\sqrt{ }$ & $\times$ & 52.8 & 60.6 \\
\hline 8 & $\sqrt{ }$ & $\times$ & $\times$ & $\sqrt{ }$ & $\times$ & $\sqrt{ }$ & 73.2 & 84.0 \\
\hline
\end{tabular}

“、”表示是, “×” 表示否, “-”表示未处理; a未经任何处理的叶片空白组;

$G$ 是一种颜色标准, $G$ 值越接近 255 , 表示颜色越接近亮绿

\section{3 数据处理方法 $[13]$}

我们用相机拍照方式记录了表 1 中 8 种条件下蔬菜叶片的最终结果以及未经任何处理的叶片空 白组, 如图 4A 和 $\mathrm{B}$ 所示。为了排除视觉判断的不准确性, 我们采用抽样调查的方法从每份蔬菜中 选取五片叶子(图 4A、B), 调查色度 ${ }^{[14]}$ 并用 PhotoShop 软件, 计算出每片叶子的 RGB ${ }^{[15]}$ 中的 $G$ 值 (一种颜色标准, $G$ 值越接近 255 , 表示颜色越接近亮绿)。最终计算得每组 $G$ 值的平均值见表 1 。

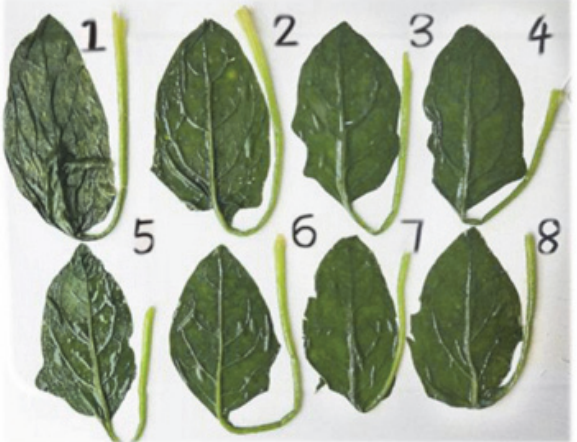

(A)

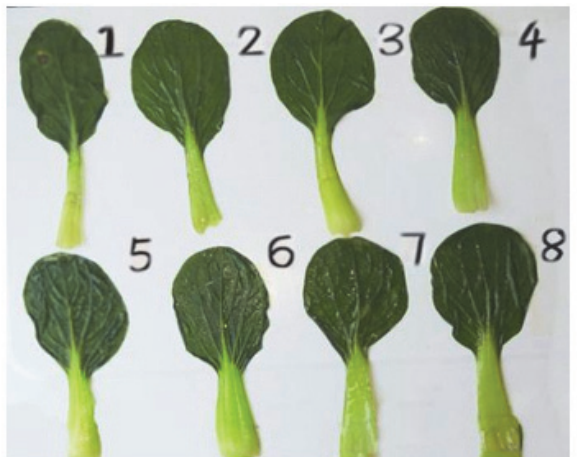

(B)

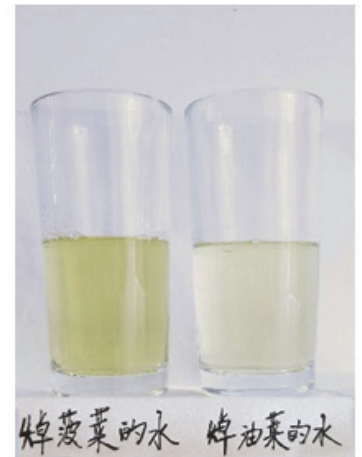

(C)

图 4 结果对比图(油滴附着会略微影响亮度)

(A) 炒好的菠菜(1-8 号)与未经任何处理的叶片空白组(0 号); (B) 炒好的油菜(1-8 号)与未经任何处理的叶片空白组 (0 号);

(C) 焯菠菜水(左)与焯油菜水(右)

相机参数：感光度 500 ; 快门速度 $1 / 100$; 手动对焦; 白平均

\section{4 结果讨论}

从表 1 可以看出, 未经任何处理的菠菜空白组平均 $G$ 值分别为新鲜样是 87.5 , 不新鲜样是 78.2 。 选用不新鲜的菠菜 1 号对照组, 平均 $G$ 值仅为 23.0 , 而选用新鲜菠菜 2 号组, $G$ 值平均为 46.8 , 说 明选用新鲜蔬菜至关重要。单独加盐的 3 号样品 $G$ 值为 48.2 , 和 2 号样品没有明显区别, 而单独加 
油的色度提高明显, 达到了 54.4, “保绿” 效果较为明显, 但是油盐同时加入的保护效果更加显著, $G$ 值达到了 64.4, 说明盐对油的 “保绿” 有明显的增效作用。单独加碱的效果和单独加油的 “保绿” 效果接近。同时加盐油焯水和炒时不盖锅盖的 “保绿” 效果最好, $G$ 值达到了 73.2, 接近空白组平 均 $G$ 值 87.5 , 也说明炒菜时不加盖子有助于 “保绿”。

油菜组的测试展示了相同的规律, 只是 $G$ 值明显都高于菠菜组。我们认为与油菜叶相比菠菜叶 片较薄, 在同样的实验条件下, 菠菜叶绿素的破坏和流失更加严重。因此, 我们也比较了焯菠菜和 油菜水的颜色(图 4C), 菠菜水的颜色明显更深, 印证了我们的猜测。同时这也提醒我们, 为了避免 叶绿素的流失, 菠菜等叶片较薄的蔬菜焯水时间不能过长。

为了不幸负这些可爱的食材, 我们艰难地 “消灭了” 这些蔬菜, 结果发现 5 号和 8 号实验组不 仅有较好的保绿效果, 同时口感也有提升。

\section{6 结语}

其实，青菜褪绿仅仅是蔬菜变色现象的 “冰山一角”。蔬菜水果变色现象在生活中也相当常见。 比如, 土豆中的多酚类物质在酶的作用下被空气氧化变成黑色; 我们常吃的紫甘蓝, 遇到不同 $\mathrm{pH}$ 溶 液会变成不同的颜色。话不多说, 小伙伴们, 疫情闭关修炼期间, 你们在家发现了什么有趣的现象, 你又是怎么用科学方法在家验证的呢, 快来一起秀一下吧!

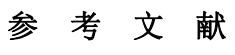

[1] 赵卫光, 关英. 大学化学, 2020, 35 (5), 256.

[2] 陈文华. 农业考古, 2005, No. 1,8.

[3] 张雪松, 张立亚, 文莲莲, 崔路兵, 侯倩, 梁芳, 张天柱, 郝天民. 蔬菜, 2019, No. 10, 36.

[4] 史天兰. 常州工学院学报(社科版), 2016, 34 (6), 84.

[5] 潘瑞炽. 植物生理学. 第 7 版. 北京: 高等教育出版社, 2012: 74-75, 78.

[6] 林厦菁, 朱晓䚲, 江青艳, 束刚. 动物营养学报, 2012, 24 (10), 1866.

[7] 王修顺, 李杰, 邱念伟. 生物学教学, 2017, 42 (9), 70.

[8] 胡明波. 叶绿素类卟啉化合物离子识别功能的研究[D]. 济南: 山东师范大学, 2009.

[9] 卢东昱, 崔新图, 黄镜荣, 赵福利. 大学物理, 2006, No. 1, 50.

[10] 约恩·博尔奇·瑟, 蒂娜·约恩森, 夏洛特·霍斯曼斯·波尔森, 马苏德·拉贾比·扎尔加海, 延斯·弗里斯贝克·瑟伦森, 詹妮·布伦斯特德, 勒内·米 凯尔森, 苏珊·M·马德里德. 用于处理含有焦脱镁叶绿素的组合物的方法: 中国, CN102803479A[P]. 2012-11-28.

[11] 王宝山主编. 植物生理学. 第 2 版. 北京: 科学出版社, 2007: 69.

[12] 鞠福明. 中国烹杄研究, 1999, No. 2, 18 .

[13] 董志强, 刘丰俊, 翁玉华, 潘莣, 颜长明, 许振玲, 张春艳, 吕银云, 欧阳小清, 阮婵姿, 任艳平. 大学化学, 2020, in press. doi: 10.3866/PKU.DXHX202005041

[14] 赵中一, 金继红. 大学化学, 2010, 25 (4), 84.

[15] 杨康叶, 邬春学. 计算机系统应用, 2013, 22 (3), 128. 